\title{
Mathematical Analysis of Eco-System Stability of Honeybee Colony Infected by Virus
}

\author{
S.A. Adebayo, S. O. Adewale \\ Department of Pure and Applied Mathematics, Ladoke Akintola University of Technology, PMB 4000, Ogbomoso, Nigeria
}

\begin{abstract}
An eco-epidemiological model interaction between hive and forager honeybee with consideration of varroa mite diseases spread in the ecosystem is represented. The model is governed by a new five-dimensional nonlinear system of ordinary differential equations to investigate the dynamics of the honeybee colony. The well-posedness of the model is established concerning the positivity and boundedness of solutions. The basic reproduction number $\left(R_{0}\right)$ was also computed, and a sensitivity analysis was carried out on $R_{0}$. The stability of the equilibrium points was determined using the Jacobian matrix with the Routh-Hurwitz criterion. Additionally, numerical simulations were performed to validate the result of the recovery class and analyzed the effect of social inhibition and disinfestation on an infected hive honeybee population in an eco-epidemiological model.
\end{abstract}

Keywords: Epidemiology, Stability, Recovered class, Disinfestation, Effect of social inhibition, Sensitivity

\section{INTRODUCTION}

$\mathrm{T}$ he interaction between organisms of different species such as the honeybee and varroa mite is an intrinsic feature of the ecosystem. Honeybee and varroa mite interaction is the study of living organisms that are subject to infestation or attack by their natural enemies acting either directly as predators, or indirectly, by disturbing the life of the colony in various ways in which the honeybees of the genus Apis are no exception (MAAREC). The honeybee is a member of the genus Apis which produces and stores honey and constructs perennial and colonial nests from wax. The best-known species of the genus is the Western honeybee (Apis mellifera, also called European or common honeybee) which was domesticated for honey production and crop pollination or at least exploited for honey and beeswax at least since the time of the building of the Egyptian pyramids (Smith et al 2000).

Colony collapse disorder (CCD) is the phenomenon in which the majority of worker bees in a colony disappear and leave behind a queen, plenty of food, and some nurse bees to care for the remaining immature bees. The phenomenon was renamed CCD in 2006 when abnormally high die-offs (30-70\% of hives) of common honeybee colonies have occurred in North America and at first, no explanation could be given (Genersch et al 2010). Several European countries have experienced the same phenomenon since 1998, and in the past few years, countries in Africa and Asia have also become affected by it. The reason seems to be a combination of factors, possibly including neonicotinoid pesticides or Israeli acute paralysis virus (Staveley et al 2014). The collapse of honeybee colonies has become widespread in several regions of the world and has been the subject of much discussion and research in recent years (vanEngelsdorp et al 2009, Ho et al 2007).

Another cause of CCD is an external parasitic mite called Varroa mite. This mite attacks the honeybees Apis cerana and Apis mellifera by attaching to the body of the bee and weakens the bee by sucking fat bodies (Ramsey et al 2019). A significant mite infestation can lead to the death of a honeybee colony and usually occurred in the late (rainy, dry, etc.). The Varroa mite is the parasite with the most pronounced economic impact on the beekeeping industry. Also, it is considered to be one of the multiple stress factors contributing to the higher levels of bee losses around the world (Goulson et al 2015). Varroa mites are carriers for many viruses that are damaging to bees, including Kashmir bee virus, sac-brood virus, acute bee paralysis virus, Israeli acute paralysis virus, and deformed wing virus (Rosenkranz et al 2010).

Several methods of treatment are currently applied to control these mites, which can be divided into chemical and mechanical controls. Usual chemical controls include "hard" synthetic chemicals such as amitraz, fluvalinate, and coumaphos, while "soft" chemical controls (organic acids, essential oils) include thymol, sucrose octanoate esters oxalic acid, and formic acid. Mechanical controls are usually based on disruption of some aspect of the mites' life cycle and they are generally intended not to eliminate all mites, but to keep the infestation at a tolerable level. Examples of mechanical controls include the sacrifice of drone brood as Varroa mites most commonly attach to the drone brood, powdered sugar dusting which encourages cleaning behavior and dislodges part of the mites, screened bottom boards which allow dislodged mites to fall through the bottom and away from the colony, brood interruption, application of heat to isolated brood combs or whole colonies and downsizing of the brood cell size. Another possibility in fighting the infestations is breeding more resistant colonies: several families of bees can coexist with Varroa mites (e.g. Africanized bees and Russian honeybees show a higher natural resistance against mites (Rosenkranz et al 2010, Buchler et al 2010)).

In this work, we incorporate five-dimensional honeybees and varroa mite model to gain further insights into the dynamics of eco-epidemiological interaction on hive and forager with the spread of the virus on the honeybee colony. The analysis of the model is extended to investigate the impact of the recovery class, namely as an effect of social inhibition and disinfestation on a hive honeybee species from infection and infestation in the ecosystem model. The rest of the work is 
organized as follows: The model is formulated and its wellposedness is established in Section 2. In Section 3, the existence and stability of possible equilibria including a derivation of the basic reproduction number are investigated. In Section 4, the sensitivity analysis and numerical simulations of the model with graphical illustrations and their discussion, Section 5 is devoted to concluding remarks.

\section{MODEL FORMULATION}

Our mathematical model is based on the presence of a mite species which is a vector for a disease as well and transmitted to a susceptible host only upon adequate contact with an infected host.

Following Dénes and Mahmoud (2019), which proposed the following model to study global dynamics of mathematical model for a honeybee colony infected by varroa mite disease. The model is given below

$$
\begin{aligned}
H_{s}^{\prime}= & A-\beta_{1} H_{s}(t) H_{m}(t)-\beta_{2} H_{s}(t) H_{i}(t)+\alpha H_{m}(t)+\alpha H_{i}(t) \\
& -d H_{s}(t)-R\left(H_{s}, F_{s}\right) H_{s}(t)+R\left(H_{s}, F_{s}\right) F_{s}(t)
\end{aligned}
$$$$
F_{s}^{\prime}=R\left(H_{s}, F_{s}\right) H_{s}(t)-\beta_{1} F_{s}(t) H_{m}(t)-\beta_{2} F_{s}(t) H_{i}(t)-d F_{s}(t)-R\left(H_{s}, F_{s}\right) F_{s}(t)
$$$$
H_{m}^{\prime}=\beta_{1} H_{s}(t) H_{m}(t)+\beta_{1} F_{s}(t) H_{m}(t)-\beta_{2} H_{m}(t) H_{i}(t)-\alpha H_{m}(t)-d H_{m}(t)
$$$$
H_{i}^{\prime}=\beta_{2} H_{s}(t) H_{i}(t)+\beta_{2} F_{s}(t) H_{i}(t)+\beta_{2} H_{m}(t) H_{i}(t)-\alpha H_{i}(t)-d H_{i}(t)-\delta H_{i}(t)
$$

Based on the above model we propose the new model for the study of the honeybee population which is divided into five compartments depending on the presence of the vector and the disease transmitted by them as follows.

i. Susceptible: those who can be infested by the vector. The healthy bee population is divided into hive bees and foragers. We denote by $M$ the compartment of susceptible hive bees and the compartment of susceptible forager bees.

ii. $P$ denotes hive bees infested by non-infectious vectors.

iii. $Q$ denotes hive bees infested by infectious vectors and thus infected with the disease.

iv. $R$ denotes the hive honeybee that recovered from infection and infestation.

The model is then formulated as follows:

$$
\begin{aligned}
& \frac{d M(t)}{d t}=A_{1}-\beta_{1} M(t) P(t)-\beta_{2} M(t) Q(t)-d M(t)-S M(t) \\
& \frac{d N(t)}{d t}=A_{2}-\beta_{1} N(t) P(t)-\beta_{2} N(t) Q(t)-d N(t)-S N(t)
\end{aligned}
$$

$$
\begin{aligned}
& \frac{d P(t)}{d t}=\beta_{1} M(t) P(t)+\beta_{1} N(t) P(t)-\beta_{2} P(t) Q(t)-\alpha P(t)-d P(t) \\
& \frac{d Q(t)}{d t}=\beta_{2} M(t) Q(t)+\beta_{2} N(t) Q(t)+\beta_{2} P(t) Q(t)-\alpha Q(t)-d Q(t)-\delta Q(t)
\end{aligned}
$$

$\frac{d R(t)}{d t}=S M(t)+S N(t)+\alpha P(t)+\alpha Q(t)-d R(t)$

Where $A_{1}$ is the natural birth rate of hive bees and $A_{2}$ immigration rate of forager bees, then $A=A_{1}+A_{2}$

Where the term $S$ represents the effect of social inhibition on the recruitment rate and is formulated as

$$
S=\sigma_{1}-\sigma_{2} \frac{N}{M+N}
$$

Where the parameter is the maximum rate at which hive bees develop into foragers when there are no foragers present in the colony. The term represents social inhibition, that is, the process whereby a surplus of foragers causes the foragers to revert to being hive bees. We assume that social inhibition is directly proportional to the forager population present in the colony.

In our model, it was assumed that the following assumptions hold.

1. Hive bees (from $P$ ) infested by non-infectious vectors can transmit the disease to susceptible (hive and forager bees), hive bees infested by infectious vectors (from $Q$ ) can transmit the disease to susceptible (hive and forager bees).

2. It was assumed that once infected or infested, forager bees are forced to stop their foraging duties because of the infestation or infection and become hive bees.

3. A hive-bee infested by infectious vectors can transmit the infection to a hive bee infested by noninfectious vectors, i.e. a member of the compartment can move to a compartment upon adequate contact with an individual from the compartment $Q$.

4. Suppose, upon adequate contact with a susceptible bee, an individual $P$ can transmit the (noninfectious) mites carried by it at the same rate to susceptibles ( $M$ and $N$ ), and we donate the transmission rate for non-infectious vectors $P$ to susceptibles by $\beta_{1}$.

5. It was assumed that, upon adequate contact with a susceptible bee or a bee with non-infectious parasites, an individual can transmit the mite carrying the virus at the same rate to susceptibles ( $M$ and $N$ ) and to those who are already infested by 
non-infectious vectors. We denote this transmission rate by $\beta_{2}$.

6. It was assumed that the disinfestation rates from the compartments to the susceptible compartment $M$ are the same and denoted by $\alpha$.

7. It was denoted by the natural birth rate of healthy hive bees and by the death rate for the compartments.

8. It was assumed that only infected hive bees due to the mites transmitting viral infection. So, we assume that the death rate of infected hive bees $Q$ is equal to $d+\delta$ where $\delta$ is the death rate caused by mites.

9. By (Betti et al., 2014), it was assumed that the proportion of recruitment of susceptible hive bees to become forager bees and the rate of healthy forager bees that are reverting to hive duties following social inhibition has the same proportion $\mathrm{S}$. We formulate this process of recruitment and social inhibition as a Holling-type II functional response, see (Ratti et al., 2017, David et al., 2011, 2013).

Table 1: Variables and parameters of the model (2.2)

\begin{tabular}{|c|c|}
\hline Variables & Description \\
\hline$M(t)$ & Susceptible hive bees \\
\hline$N(t)$ & Susceptible forager bees \\
\hline$P(t)$ & $\begin{array}{l}\text { Hive bees infested by non- } \\
\text { infectious vectors }\end{array}$ \\
\hline$Q(t)$ & $\begin{array}{l}\text { Hive bees infested by infectious } \\
\text { vectors }\end{array}$ \\
\hline$R(t)$ & Recovered/Removed hive bees \\
\hline$T(t)$ & Total Population of honeybee \\
\hline Parameter & Description \\
\hline$A_{1}$ & The natural birth rate of hive bees \\
\hline$A_{2}$ & $\begin{array}{c}\text { The immigration rate of forager } \\
\text { bees }\end{array}$ \\
\hline$\beta_{1}$ & $\begin{array}{l}\text { The transmission rate for non- } \\
\text { infectious vectors }\end{array}$ \\
\hline$\beta_{2}$ & $\begin{array}{c}\text { Transmission rate infested by non- } \\
\text { infectious vectors }\end{array}$ \\
\hline$\delta$ & Death rate caused by mites \\
\hline$d$ & Natural death rate \\
\hline$\alpha$ & Disinfestation rate \\
\hline$S$ & Social inhibition \\
\hline
\end{tabular}

\subsection{Well-posedness of the model}

The mathematical and eco-epidemiological relevance of the honeybee-varroa mite system depends on the well-posedness of the model. Keep in mind that all the parameters of the model are non-negative. Here, the boundedness and positivity of solutions of the model are investigated to establish the well-posedness of the model.

\subsubsection{Boundedness of solutions}

The following result is required to establish the boundedness of solutions of the model (2.2).

Theorem 2.2: Every solution in the solution set of the model given by (2.2) is positively invariant and attracting. This implies that every solution approaches and remains in the solution set as.

Proof:

Let the total model (2.2) be represented, respectively $T(t)=M(t)+N(t)+P(t)+Q(t)+R(t)$

Then it follows that

$$
\frac{d T(t)}{d t} \leq A-d T(t)
$$

Solving the nonlinear first-order differential inequality of Bernoulli type (2.2) yields

$$
T(t) \leq \frac{A}{d}+A\left(T(0)-\frac{A}{d}\right) e^{-d t}
$$

As $T \rightarrow \infty, T(t) \rightarrow \frac{A}{d}$

This implies that $0 \leq T(t) \leq \frac{A}{d}$ at any time $t \geq 0$, and so every solution with initial conditions in $\mathfrak{R}_{+}^{5}$ approaches remains in the region for all $t \geq 0$.

$\begin{array}{lr}\text { Hence, } & \text { region } \\ \Omega=\left\{M(t), N(t), P(t), Q(t), R(t) \in \mathfrak{R}_{+}^{5}: T(t) \leq \frac{A}{d}\right\} \text { is }\end{array}$ positively invariant and attracting. This end ends the proof.

\subsubsection{Positivity of solutions}

Theorem 2.2: The solution set of the eco-epidemiological model (2.2) with non-negative initial conditions and remains non-negative for all time $\mathrm{t}>0$.

Proof: The first equation of the model (2.2), implies that

$$
\frac{d M}{d t}+\left\{\left(\beta_{1} P(t)+\beta_{2} Q+d+S\right)\right\} M \geq 0
$$

Which on using integrating factor yields

$$
M(t) \exp \left[\int_{0}^{t}\left(\beta_{1} P(\theta) d \theta+\beta_{2} Q(\theta)\right) d \theta+(d+S) t\right] \geq M(0)
$$

Further integration of (2.6) yields 
$M(t) \geq M(0) \exp \left[-\int_{0}^{t}\left(\beta_{1} P(\theta) d \theta+\beta_{2} Q(\theta)\right) d \theta+(d+S) t\right]>0, \forall t>0$

All other variables can be proved to be positive in a similar approach.

Hence, we can say the positivity of the Solution of the model (2.2)

$\Omega=\left\{M(t), N(t), P(t), Q(t), R(t)\right.$ and $\left.T(t) \in \mathfrak{R}_{+}^{5}\right\}$ of eco-epidemiologically is well-posed.

This ends the proof

\section{ANALYSIS OF THE MODEL}

In this section, the hive and forager honeybee model is analyzed around the possible equilibrium points.

The existence of equilibrium points

The hive and forager honeybee model (2.2) has the following possible equilibrium points:

\subsubsection{Trivial equilibrium points $\left(E_{0}\right)$}

This is a steady state in the absence of honeybee colony. Hence, no interactions exist. The equilibrium point is given by

$$
E_{0}=(0,0,0,0,0)
$$

\subsubsection{Disease Free and infestation Free Equilibrium Point $\left(E_{1}\right)$}

This is simply the point where there is no existence of the disease in the honeybee colony, such that the colony has no threat of infestation. The equilibrium point is given by

$E_{1}=\left(\frac{A A_{1}\left(d+\sigma_{1}\right)-\sigma_{2} A_{1} A_{2}}{A}, \frac{A A_{2}\left(d+\sigma_{1}\right)-\sigma_{2} A_{2}^{2}}{A}, 0,0,\left(\sigma_{2} d+\sigma_{1} \sigma_{2}\right)\left(A-A_{2}\right)-A_{2} \sigma_{2}^{2}\left(1-\frac{A_{2} d}{A}\right)\right)$

\subsubsection{Disease Free with Infestation equilibrium Point $E_{2}$}

This is a steady state where there is no form of the disease with infestation. Here, the honeybee colony lives freely without fear of infestation while the infection is absent in the colony. The equilibrium point is given as $E_{2}=\left(\begin{array}{l}\frac{(\alpha+d+\delta) A_{1}}{\beta_{2} A}, \frac{(\alpha+d+\delta) A_{2}}{\beta_{2} A}, 0, \frac{A \beta_{2}-\left(A d+A \sigma_{1}-A_{2} \sigma_{2}\right)(\alpha+d+\delta)}{A \beta_{2}(\alpha+d+\delta)}, \\ \frac{(\alpha+d+\delta)\left[\left(\sigma_{1} A-\sigma_{2} A_{2}\right)(\alpha+d+\delta)-\alpha\left(A d+A \sigma_{1}-A_{2} \sigma_{2}\right)\right]+A^{2} \beta_{2} \alpha}{A d \beta_{2}(\alpha+d+\delta)}\end{array}\right)$

\subsubsection{Endemic and Infestation with Infectious Vectors} Equilibrium Point

This is the state when there is endemic and infestation with infections vectors in the colony. Here, the honeybee colony is already affected by an infection. The equilibrium point is given by

$$
E_{3}=\left(\frac{A_{1}(\alpha+d)}{\beta_{1} A}, \frac{A_{2}(\alpha+d)}{\beta_{1} A}, \frac{A \beta_{1}-(d+S)(\alpha+d)}{(\alpha+d) \beta_{1}}, 0, \frac{A\left(\sigma_{1}-\alpha\right)-\sigma_{2} A_{2}+\alpha A^{2} \beta_{2}}{A \beta_{2} d(\alpha+d)}\right)
$$

\subsubsection{Endemic and Infestation with Infectious and Non-} Infectious Vectors Equilibrium Point

This is the state when there is an endemic and infestation with infections a non-infectious vector in the colony. The equilibrium point is given by

$$
E_{4}=\frac{A_{1} \beta_{2}^{2}\left\lfloor A\left(\sigma_{1}-\alpha\right)-A_{2} \sigma_{2}\right\rfloor+A A_{1} \beta_{1} \beta_{2}(\alpha+d+\delta)}{A}, \frac{A_{2} \beta_{2}^{2}\left\lfloor A\left(\sigma_{1}-\alpha\right)-A_{2} \sigma_{2}\right\rfloor+A A_{2} \beta_{1} \beta_{2}(\alpha+d+\delta)}{A},
$$

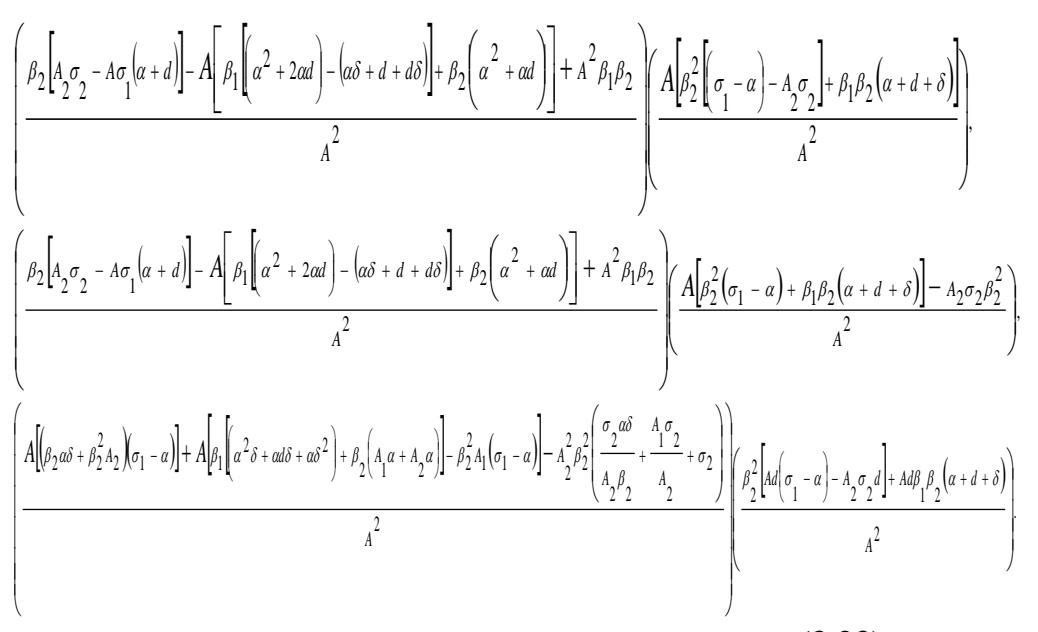

\subsection{Stability analysis}

The Jacobian matrix of the honeybee and varroa mite system (2.2) is given by

$J=\left(\begin{array}{ccccc}J_{1} & 0 & -\beta_{1} M & -\beta_{1} M & 0 \\ 0 & J_{2} & -\beta_{1} N & -\beta_{2} N & 0 \\ \beta_{1} P & \beta_{1} P & J_{3} & -\beta_{2} P & 0 \\ \beta_{2} Q & \beta_{2} Q & \beta_{2} Q & J_{4} & 0 \\ S & S & \alpha & \alpha & -d\end{array}\right)$

Where

$$
\begin{aligned}
& J_{1}=-\beta_{1} P-\beta_{2} Q-d-S \\
& J_{2}=-\beta_{1} P-\beta_{2} Q-d-S \\
& J_{3}=\beta_{1} M P+\beta_{1} N-\beta_{2} Q-\alpha-d \\
& J_{4}=\beta_{2} M+\beta_{2} N+\beta_{2} P-\alpha-d-\delta
\end{aligned}
$$

The stability of each of the equilibrium points is analyzed by 
finding the eigenvalues of the Jacobian matrix (3.24) evaluated at each point.

\subsubsection{Stability of $E_{0}$}

The Jacobian matrix (3.24) is evaluated at the trivial equilibrium point (3.19). Hence, solving the corresponding characteristic equation $\left.\mid J\left(E_{0}\right)-\lambda I 5\right) \mid=0$, where $\lambda$ is the eigenvalue and $\mathrm{I} 5$ is the identity matrix of order five, the following eigenvalues are obtained:

$\lambda_{1,2}=-\left(\frac{d A+A \sigma_{1}-\sigma_{2} A_{2}}{A}\right)$,

$\lambda_{3}=-(\alpha+d), \quad \lambda_{4}=-(\alpha+d+\delta), \lambda_{5}=-d$

Now, all the eigenvalues are negative, if $\left(d A+\sigma_{1} A\right)>\sigma_{2} A_{2}$. Hence, the trivial equilibrium $E_{0}$ is locally asymptotically stable, provided that $\left(-d A-\sigma_{1} A\right)>\sigma_{2} A_{2}$.

\subsubsection{Stability of $E_{1}$}

The characteristic equation of the Jacobian matrix (3.24) evaluated at the disease-free and infestation free equilibrium point $E_{1}$ is given by $\left.\mid \mathrm{J}\left(\mathrm{E}_{1}\right)-\lambda \mathrm{I} 5\right) \mid=0$, which gives the following eigenvalues:

$$
\begin{aligned}
& \lambda_{1,2}=-\left(d+\sigma_{1}-\frac{\sigma_{2} A_{2}}{A}\right), \\
& \lambda_{3}=-\left[(\alpha+d)+\beta_{1}\left(A\left(\sigma_{1}+d\right)-\sigma_{2} A_{2}\right)\right] \\
& \lambda_{4}=-(\alpha+d+\delta), \quad \lambda_{5}=-d
\end{aligned}
$$

Now, all the eigenvalues are negative, if $d+\sigma_{1}>-\frac{\sigma_{2} A_{2}}{A}$ and $(\alpha+d)+\beta_{1} A\left(\sigma_{1}+d\right)>-\sigma_{2} A_{2} \beta_{1}$. Hence, the Disease and infestation free equilibrium $E_{1}$ is locally asymptotically stable, provided that $-d-\sigma_{1}>\frac{\sigma_{2} A_{2}}{A}$ and $-(\alpha+d)-\beta_{1} A\left(\sigma_{1}+d\right)>\sigma_{2} A_{2} \beta_{1}$

\subsubsection{Stability of $E_{2}$}

The characteristic equation of the Jacobian matrix (3.24) evaluated at the disease-free infestation equilibrium point $E_{2}$ is given by $\left.\mid J\left(\mathrm{E}_{2}\right)-\lambda \mathrm{I} 5\right) \mid=0$, which gives the following eigenvalues:

$$
\begin{aligned}
& \lambda_{1}=-V_{2}, \lambda_{2}=-d, \quad \lambda_{3}=-V \\
& \lambda_{4}=\frac{V+\sqrt{V^{2}+4 V_{3} V}}{2}, \lambda_{5}=\frac{V-\sqrt{V^{2}+4 V_{3} V}}{2}
\end{aligned}
$$

Not all the eigenvalues are negative. Hence, the disease-free with infestation equilibrium point $E_{2}$ is unstable.

\subsubsection{Stability of $E_{3}$}

The characteristic equation of the Jacobian matrix (3.24) evaluated at the endemic and infestation with infectious vectors equilibrium point $E_{3}$ is given by $\left.\mid \mathrm{J}\left(\mathrm{E}_{3}\right)-\lambda \mathrm{I} 5\right) \mid=0$, which gives the following eigenvalues:

$$
\begin{aligned}
& \lambda_{1}=U_{6}, \quad \lambda_{2}=-d, \quad \lambda_{3}=U, \\
& \lambda_{4}=\frac{\left(U+U_{4}\right)+\sqrt{\left(U+U_{4}\right)^{2}-4\left(U U_{4}-U_{3} U_{1}\right)}}{2}
\end{aligned}
$$

$\lambda_{5}=\frac{\left(U+U_{4}\right)-\sqrt{\left(U+U_{4}\right)^{2}-4\left(U U_{4}-U_{3} U_{1}\right)}}{2}$

Not all the eigenvalues are strictly negative. Hence, the endemic and infestation with infectious vectors equilibrium point $E_{3}$ are unstable.

\subsubsection{Stability of $E_{4}$}

The Jacobian matrix (3.24) is evaluated at the endemic and infestation with infectious and non-infectious vectors equilibrium point $E_{4}$ hence, solving the corresponding characteristic equation $\left.\mid J\left(E_{4}\right)-\lambda I 5\right) \mid=0$ gives one eigenvalue and the remaining four eigenvalues are obtainable from

Where

$$
\begin{aligned}
& W_{2}=-\beta_{1}\left(A_{1} \beta_{2}^{2}\left(\sigma_{1}-\alpha\right)+A_{1} \beta_{1} \beta_{2}(\alpha+d+\delta)-\frac{A_{1} A_{2} \sigma_{2} \beta_{2}^{2}}{A}\right) \\
& W_{3}=-\beta_{1}\left(A_{2} \beta_{2}^{2}\left(\sigma_{1}-\alpha\right)+A_{2} \beta_{1} \beta_{2}(\alpha+d+\delta)-\frac{A_{2}^{2} \sigma_{2} \beta_{2}^{2}}{A}\right)
\end{aligned}
$$

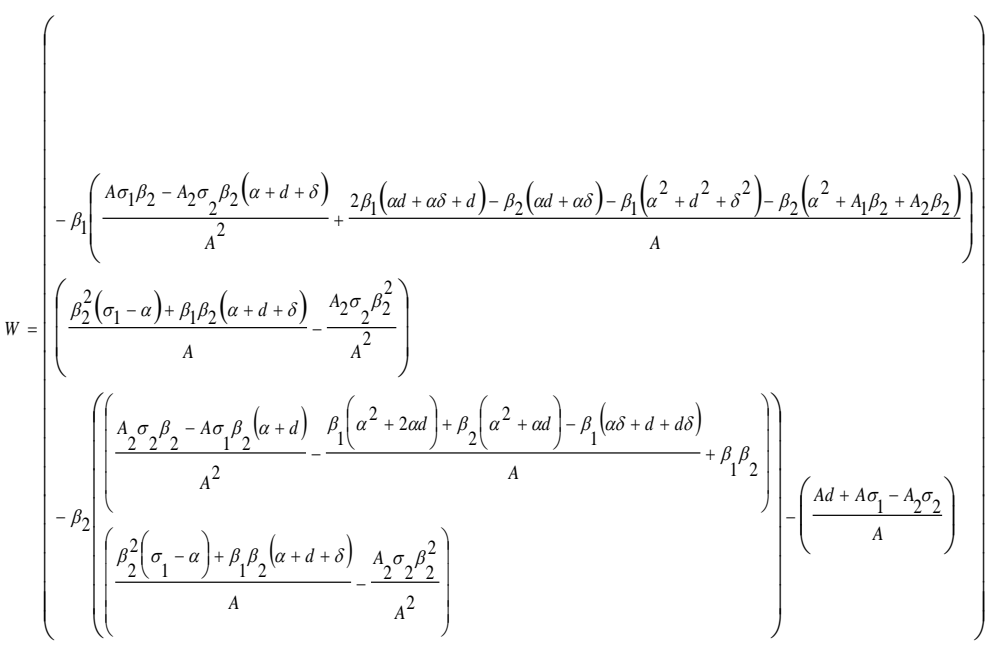




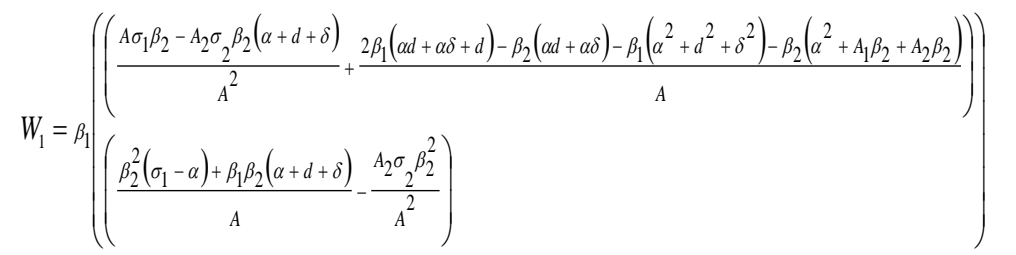

$\left(\beta_{1}\left(\begin{array}{l}\frac{A \sigma_{1} \beta_{2}-A_{2} \sigma_{2} \beta_{2}(\alpha+d+\delta)}{A^{2}} \\ +\frac{2 \beta_{1}(\alpha d+\alpha \delta+d)-\beta_{2}(\alpha d+\alpha \delta)-\beta_{1}\left(\alpha^{2}+d^{2}+\delta^{2}\right)-\beta_{2}\left(\alpha^{2}+A_{1} \beta_{2}+A_{2} \beta_{2}\right)}{A}\end{array}\right)\right.$

$\left(\frac{\beta_{2}^{2}\left(\sigma_{1}-\alpha\right)+\beta_{1} \beta_{2}(\alpha+d+\delta)}{A}-\frac{A_{2} \sigma_{2} \beta_{2}^{2}}{A^{2}}\right)\left(A_{1} \beta_{2}^{2}\left(\sigma_{1}-\alpha\right)+A_{1} \beta_{1} \beta_{2}(\alpha+d+\delta)-\frac{A_{1} A_{2} \sigma_{2} \beta_{2}^{2}}{A}\right)$

$W_{4}=+\beta_{1}\left(A_{2} \beta_{2}^{2}\left(\sigma_{1}-\alpha\right)+A_{2} \beta_{1} \beta_{2}(\alpha+d+\delta)-\frac{A_{2}^{2} \sigma_{2} \beta_{2}^{2}}{A}\right)$

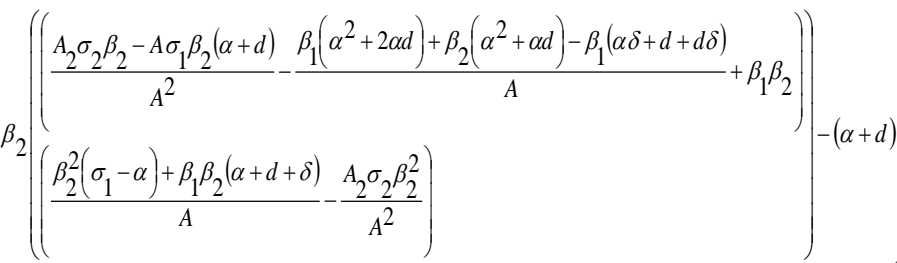

$W_{5}=\beta_{2}\left(\begin{array}{l}\left(\frac{A_{2} \sigma_{2} \beta_{2}-A \sigma_{1} \beta_{2}(\alpha+d)}{A^{2}}-\frac{\beta_{1}\left(\alpha^{2}+2 \alpha d\right)+\beta_{2}\left(\alpha^{2}+\alpha d\right)-\beta_{1}(\alpha \delta+d+d \delta)}{A}+\beta_{1} \beta_{2}\right) \\ \left(\frac{\beta_{2}^{2}\left(\sigma_{1}-\alpha\right)+\beta_{1} \beta_{2}(\alpha+d+\delta)}{A}-\frac{A_{2} \sigma_{2} \beta_{2}^{2}}{A^{2}}\right)\end{array}\right)$

$\left(\left(\frac{A \sigma_{1} \beta_{2}-A_{2} \sigma_{2} \beta_{2}(\alpha+d+\delta)}{A^{2}}+\frac{2 \beta_{1}(\alpha d+\alpha \delta+d)-\beta_{2}(\alpha d+\alpha \delta)-\beta_{1}\left(\alpha^{2}+d^{2}+\delta^{2}\right)-\beta_{2}\left(\alpha^{2}+A_{1} \beta_{2}+A_{2} \beta_{2}\right)}{A}\right)\right)$ $W_{6}=-\beta_{2}$

$\left(\frac{\beta_{2}^{2}\left(\sigma_{1}-\alpha\right)+\beta_{1} \beta_{2}(\alpha+d+\delta)}{A}-\frac{A_{2} \sigma_{2} \beta_{2}^{2}}{A^{2}}\right)$

$W_{7}=A_{1} \beta_{2}^{3}\left(\sigma_{1}-\alpha\right)+A_{1} \beta_{1} \beta_{2}^{2}(\alpha+d+\delta)-\frac{\sigma_{2} A_{1} A_{2} \beta_{2}^{3}}{A}+A_{2} \beta_{2}^{3}\left(\sigma_{1}-\alpha\right)+A_{2} \beta_{1} \beta_{2}^{2}(\alpha+d+\delta)-\frac{A_{2}^{2} \sigma_{2} \beta_{2}^{3}}{A}$

$+\left(\begin{array}{l}\sigma_{1} \beta_{2}^{2}(\alpha+d+\delta)-\frac{\sigma_{2} A_{2} \beta_{2}^{2}(\alpha+d)}{A}+\alpha \beta_{1} \beta_{2}(\alpha+2 d+2 \delta) \\ -\alpha \beta_{2}^{2}(\alpha+d+\delta)+\beta_{1} \beta_{2}\left(d^{2}+2 d \delta+\delta^{2}\right)-\beta_{2}^{3}\left(A_{1}+A_{2}\right)-\sigma_{2} A_{2} \delta \beta_{2}^{2}\end{array}\right)\left(\begin{array}{l}\beta_{2}^{2}\left(\sigma_{1}-\alpha\right) \\ +\beta_{1} \beta_{2}(\alpha+d+\delta)-\frac{\sigma_{2} A_{2} \beta_{2}^{2}}{A}\end{array}\right)-(\alpha+d+\delta)$

$\lambda^{4}+\lambda^{3} R_{1}+\lambda^{2} R_{2}+\lambda R_{3}+R_{4}=0$

Where

$R_{1}=2 W-W_{4}-W_{7}$

$\mathrm{R}_{2}=\mathrm{W}^{2}-2 \mathrm{WW}_{4}-2 W W_{7}+W_{4} W_{7}+W_{6} W_{5}+W_{1} W_{3}+W_{5} W_{3}+W_{1} W_{2}$
$R_{3}=W W_{1} W_{3}+W W_{5} W_{3}+W W_{1} W_{2}-W^{2} W_{4}-W^{2} W_{7}+2 W W_{4} W_{7}+2 W W_{6} W_{5}-W_{1} W_{3} W_{7}-W_{3} W_{6} W_{5}$ $+W_{1} W_{5} W_{3}-W_{5} W_{3} W_{4}-W_{1} W_{2} W_{7}+W_{1} W_{2} W_{5}-W_{2} W_{5}$

$\mathrm{R}_{4}=\mathrm{W}^{2} W_{4} W_{7}+\mathrm{W}^{2} W_{6} W_{5}-W W_{1} W_{3} W_{7}-W W_{3} W_{6} W_{5}+W W_{1} W_{5} W_{3}-W W_{5} W_{3} W_{4}-W W_{1} W_{2} W_{7}+W W_{1} W_{2} W_{5}$ $+W_{2} W_{5} W_{4}-W_{2} W_{6} W_{5}$

Applying The Routh Hurwitz criterion, the root of (3.25) will have negative real parts the Hurwitz matrices satisfy the following conditions.

All the eigenvalues are negative satisfying the Routh Hurwitz condition. Hence, the endemic and infestation with infectious and non-infectious vectors $E_{4}$ equilibrium points are locally asymptotically stable.

3.2.6 Global Stability of Disease-Free and Infestation-Free Equilibrium $\left(E_{1}\right)$

Consider the model (2) non-infective and non-infectious subsystem, it follows as:

Where, $\frac{d X}{d t}=F(X, Y)$ and of infective and infestation sub-

system $\quad \frac{d Y}{d t}=G(X, Y), G(X, 0)=0 \quad$ Where

$X=(M, N, R)$ and $Y=(P, Q)$

3.2.7 Theorem: The disease-free and infestation-free equilibrium point, $E_{1}=\left(X^{*}, 0\right)$ is globally asymptotically stable (GAS) provide the $R_{0}<1$ (i.e. $E_{1}$ is locally asymptotically stable) and the assumption and below are satisfied.

$\left(H_{1}\right)$ : For $\frac{d X}{d t}=F(X, 0), X^{*}$ is globally asymptotically stable

$\left(H_{2}\right)$ : is an M-Matrix and is the region where the model is biologically feasible.

Proof: the model (2), it follows: $X=(M, N, R)$ and $Y=(P, Q)$

$\hat{G}(X, Y)=\left(\begin{array}{l}\beta_{1} P\left[\left(M^{*}+N^{*}\right)-(M+N)\right]+\beta_{2} P Q \\ \beta_{2} Q\left[\left(M^{*}+N^{*}\right)-(M+N)\right]-\beta_{2} P Q\end{array}\right)=\left(\begin{array}{l}\hat{G}_{1}(X, Y) \\ \hat{G_{2}}(X, Y)\end{array}\right)$ (3.21)

Now, if and $\hat{G}_{2}(X, Y) \geq 0$ if $\left(M^{*}+N^{*}\right) \geq(M+N)$ and $\beta_{2} Q\left[\left(M^{*}+N^{*}\right)-(M+N)\right] \geq \beta_{2} P Q$

Then, $\hat{G}(X, Y) \geq 0$ 
Hence the disease-free and infestation-free equilibrium is globally asymptotically stable, provided that $R_{o}<1$. otherwise it is unstable.

3.2.8 Global Stability of Endemic and Infestation with Infectious and Non-Infectious Vectors Equilibrium Point $E_{4}$

3.2.9 Theorem: The equilibrium point is globally asymptotically stable if given a Lyaponov function, the time derivative of which is semi-negative definite.

Proof: Let

$$
\left.\begin{array}{l}
L=\left[M-M^{*}-M^{*} \ln \left(\frac{M}{M^{*}}\right)\right]+\left[N-N^{*}-N^{*} \ln \left(\frac{N}{N^{*}}\right)\right] \\
+\left[P-P^{*}-P^{*} \ln \left(\frac{P}{P^{*}}\right)\right]+\left[Q-Q^{*}-Q^{*} \ln \left(\frac{Q}{Q^{*}}\right)\right] \\
+\left[R-R^{*}-R^{*} \ln \left(\frac{R}{R^{*}}\right)\right]
\end{array}\right\}
$$

Taking the time derivative of, we have

$$
\frac{d L}{d t}=\left(1-\frac{M^{*}}{M}\right) \frac{d M}{d t}+\left(1-\frac{N^{*}}{N}\right) \frac{d N}{d t}+\left(1-\frac{P^{*}}{P}\right) \frac{d P}{d t}+\left(1-\frac{Q^{*}}{Q}\right) \frac{d Q}{d t}+\left(1-\frac{R^{*}}{R}\right) \frac{d R}{d t}
$$

At equilibrium, the time derivative of each class is zero. This implies that from equation model (2)

$$
\frac{d L}{d t}=-\left[\left(\frac{\left(M-M^{*}\right)^{2}}{M}+\frac{\left(N-N^{*}\right)^{2}}{N}\right)\left(\beta_{1} P+\beta_{2} Q+(d+S)\right)+\frac{d\left(R-R^{*}\right)^{2}}{R}\right]
$$

Now, the equilibrium being the globally minimum of implies that $M^{*} \leq M, N^{*} \leq N$ and $R^{*} \leq R$. Therefore from (3.51), $\frac{d L}{d t} \leq 0$ if and only if and. Thus the largest compact invariant set in is the singleton set, which is the endemic and infestation with infectious and non-infectious vectors equilibrium for honeybee with varroa mite colony. Hence, $E_{4}=\left(M^{*}, N^{*}, P^{*}, Q^{*}, R^{*}\right)$ is globally asymptotically stable in the region. This completes the prove

\subsection{Basic Reproduction Number}

Considering that there are only two disease classes in the hive bees infected by noninfectious vector and hive bees infected by infectious vector-virus system model $(2.2 \mathrm{c}-\mathrm{d})$, then using the notations in the next generation operator method, it follows that

$$
f=\left(\begin{array}{c}
\beta_{1} P(M+N)-\beta_{2} P Q \\
\beta_{2} Q(M+N)+\beta_{2} P Q
\end{array}\right) \quad V=\left(\begin{array}{c}
(\alpha+d) P \\
(\alpha+d+\delta) Q
\end{array}\right)
$$

Therefore the basic reproduction number of the honeybee colony system (2.2) is given by.

$$
R_{0}=\operatorname{Max}\left\{\left(A \beta_{1}\left(\sigma_{1}+d\right)-\sigma_{2} A_{2} \beta_{1}\right)(\alpha+d),\left(A \beta_{2}\left(\sigma_{1}+d\right)-\sigma_{2} A_{2} \beta_{2}\right)(\alpha+d+\delta)\right\}
$$

\section{SENSITIVITY ANALYSIS}

\section{Sensitivity analysis of basic reproduction number}

Eco-epidemiological sensitivity analysis is an important notion that determines the impact of each parameter on disease transmission. The normalized forward sensitivity indices of $R_{0}$ that depends differentially on a parameter $P$ is defined as

$$
\chi_{p}^{R_{0}}=\frac{\partial R_{0}}{\partial P} \times \frac{P}{R_{0}}
$$

Table 2: Sensitivity Indices of the Basic Reproduction Number

\begin{tabular}{|c|c|c|}
\hline Parameters & Baseline Values & Sensitivity Indices \\
\hline$\beta_{1}$ & $5 \times 10^{-5}$ & +1.000000 \\
\hline$A_{1}$ & 625 & +0.570175 \\
\hline$A_{2}$ & 500 & -0.048387 \\
\hline$\sigma_{1}$ & 0.25 & +1.008065 \\
\hline$\sigma_{2}$ & 0.75 & -0.048387 \\
\hline$d$ & 0.01 & +0.016129 \\
\hline$\alpha$ & 0.01 & -0.024194 \\
\hline
\end{tabular}

Sensitivity indices of $R_{0}$

Using

$R_{0}=\left[\left(A_{1}+A_{2}\right) \beta_{1}\left(\sigma_{1}+d\right)-\sigma_{2} A_{2} \beta_{1}(\alpha+d)\right]$

where

and $P$ is any given parameter. Then,

The result of the sensitivity analysis showed that maximum growth of foragers $\sigma_{1}$ and the transmission rate, $\beta_{1}$ are the most sensitive parameters to the reproduction number with positive sensitivity indices.

For example, $\chi_{\beta_{1}}^{R_{0}}=+1.000000$ means that increasing (or decreasing) $\beta_{1}$ by $10 \%$ increases (or decreases) $R_{0}$ by $10 \%$; while means that increasing (or decreasing) $\sigma_{2}$ by $10 \%$ decreases (or increases) by $0.24194 \%$. The interpretation of the sensitivity indices of other parameters follows a similar manner as that of $\beta_{1}$ and $\sigma_{1}$.

\subsection{Numerical Simulations and results}

The numerical simulation of the Eco-epidemiological model was carried out by Maple 18.0 software using direct substitution methods to show the results model of the 
equations, the global stability of the equilibria, and the effects of parameters like the effect of social inhibition $S$ disinfestation, transmission rate and recovered. We used some of the parameter values compatible with varroa mite disease as given in Table3: below, and by considering the initial conditions:

Table 3: Parameter Values used in the model

\begin{tabular}{|c|c|c|}
\hline Parameter & Value & Sources \\
\hline$A_{1}$ & 625 & {$[13]$} \\
\hline$A_{2}$ & 500 & {$[13]$} \\
\hline$\beta_{1}$ & $5 \times 10^{-5}$ & {$[2]$} \\
\hline$\beta_{2}$ & $5 \times 10^{-5}$ & {$[2]$} \\
\hline$d$ & 0.01 & {$[9]$} \\
\hline$\delta$ & 0.14 & Assumed \\
\hline$\sigma_{1}$ & 0.25 & {$[4]$} \\
\hline$\sigma_{2}$ & 0.75 & {$[4]$} \\
\hline$\alpha$ & 0.01 & Assumed \\
\hline
\end{tabular}

\subsection{Recommendation}

The efforts such as social inhibition $\left(\sigma_{2}\right)$ and disinfestation $(\alpha)$ that enhance the recovery of hive honeybee species from infection and infestation in the ecosystem should be encouraged.

\subsection{Contributions to Knowledge}

The contributions of this study to the existing body of knowledge are as follows:

1. The formulations and analysis of a new ecoepidemiological model for varroa mite disease spread in honeybee colonies by incorporating the recovery/removal of infected hive honey bees population due to immunity.

2. The establishment of conditions that will prevent the extinction of hive and forager honeybees populations in an ecosystem where there are epidemics to ensure a disease-free state.

Results

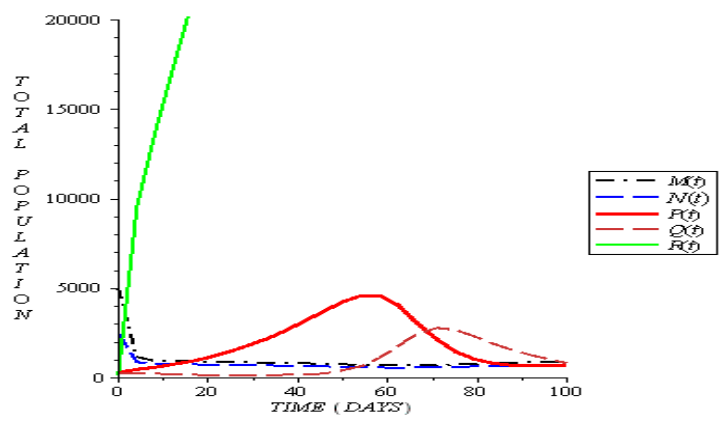

Fig. 1: Plot of the numerical solution of the model showing the behavior of all compartment

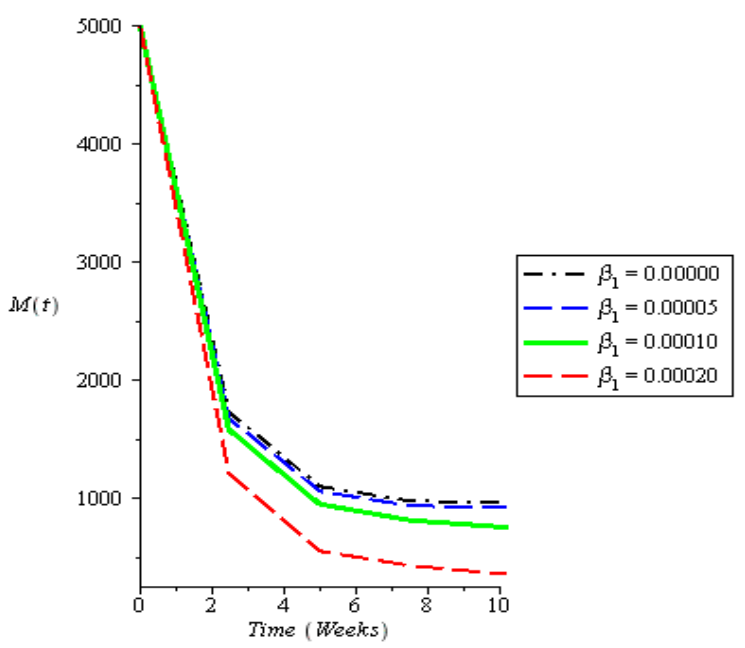

Fig. 2: Plot of the effect of transmission rate, $\beta_{1}$ on the susceptible hive

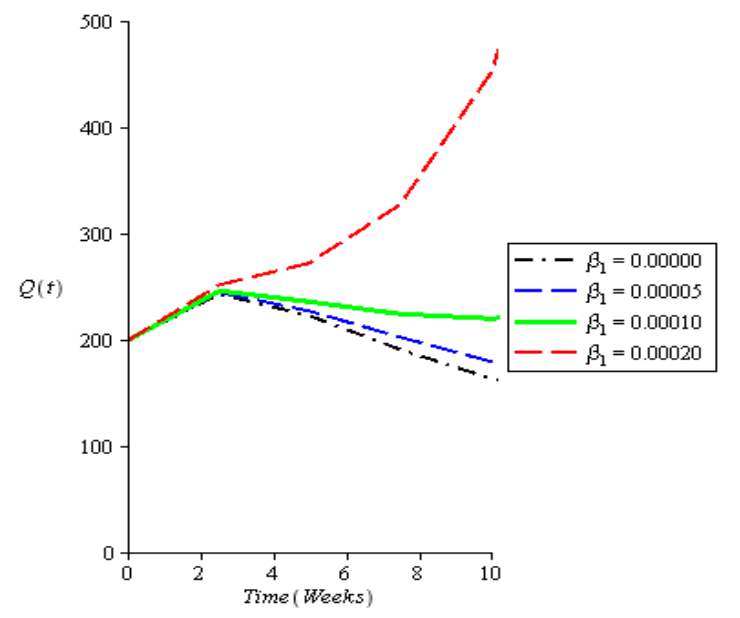

Fig. 3: Effect of transmission rate, $\beta_{1}$ on the hive bees infested by infectious vectors

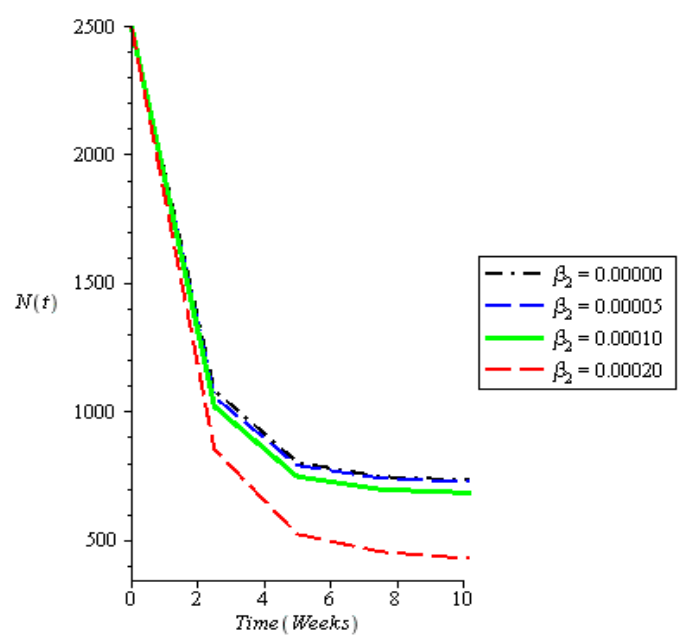

Fig. 4: Plot of the effect of transmission rate, $\beta_{2}$ on the susceptible forager bees 


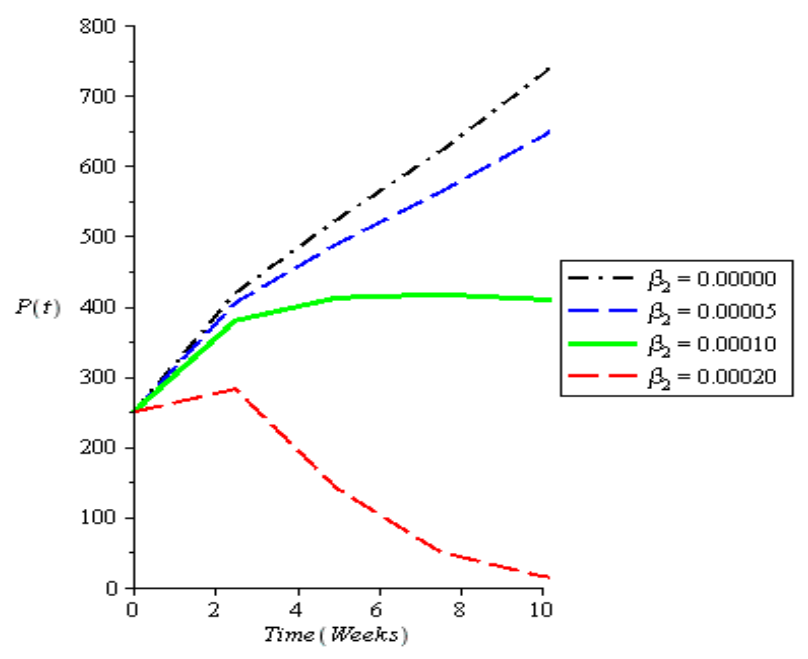

Fig. 5: Plot of the effect of transmission rate, $\beta_{2}$ on the hive bees infested by non-infectious vectors

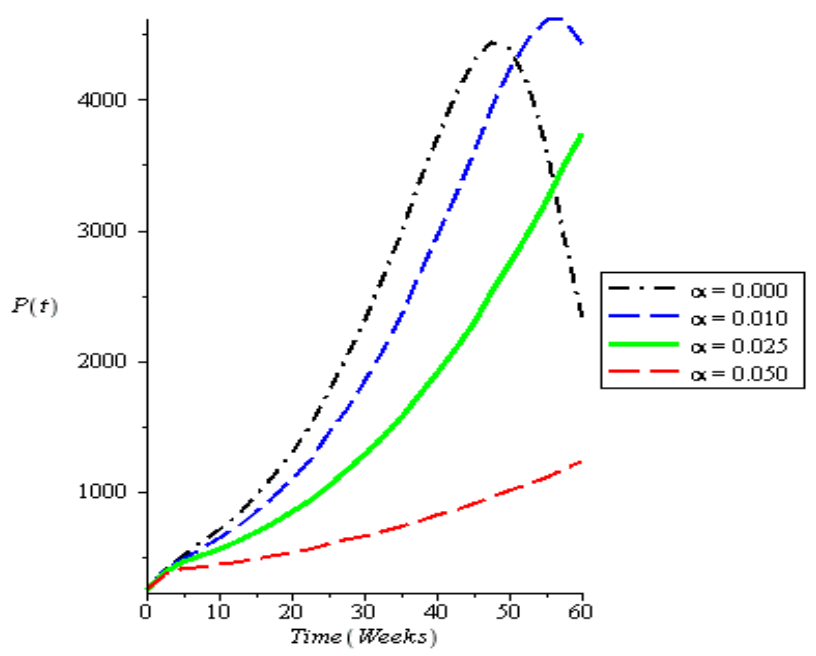

Fig. 6: Plot of the effect of disinfestation rate, $\alpha$ on the hive bees infested by non-infectious vectors

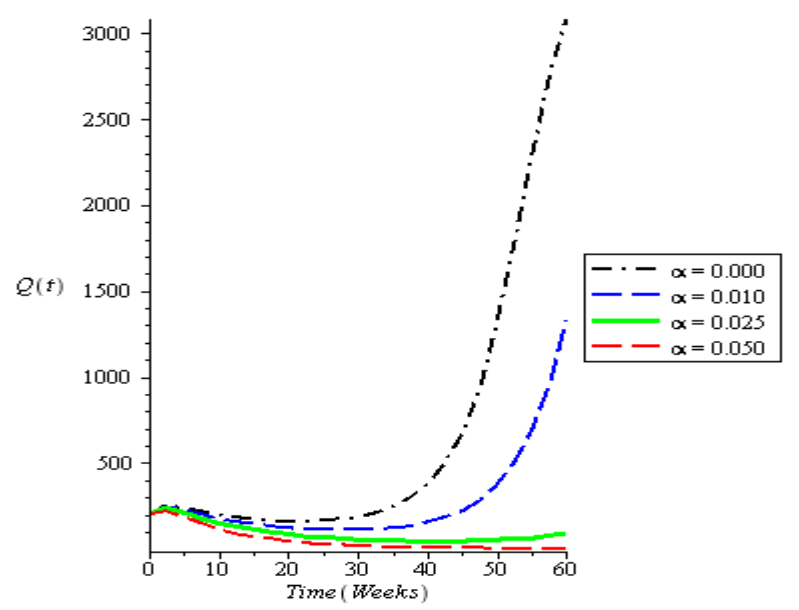

Fig. 7: Plot of the effect of disinfestation rate, $\alpha$ on the hive bees infested by infectious vectors

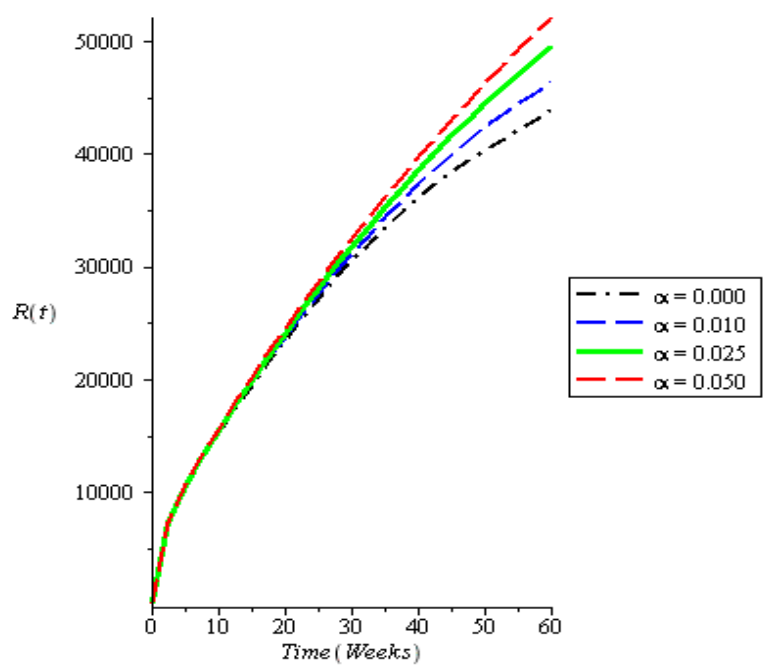

Fig. 8: Plot of the effect of disinfestation rate, $\alpha$ on the Recovered class

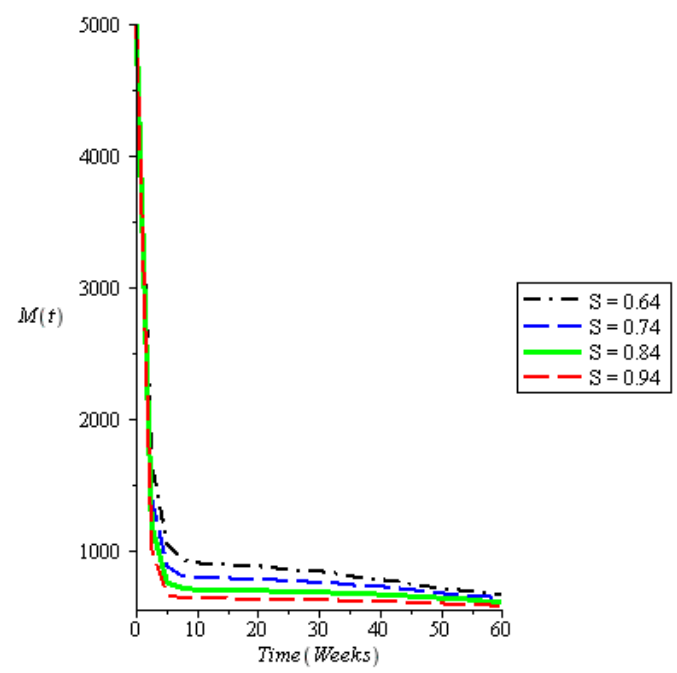

Fig. 9: Plot of the effect of social inhibition, $S$ on the susceptible hive bees

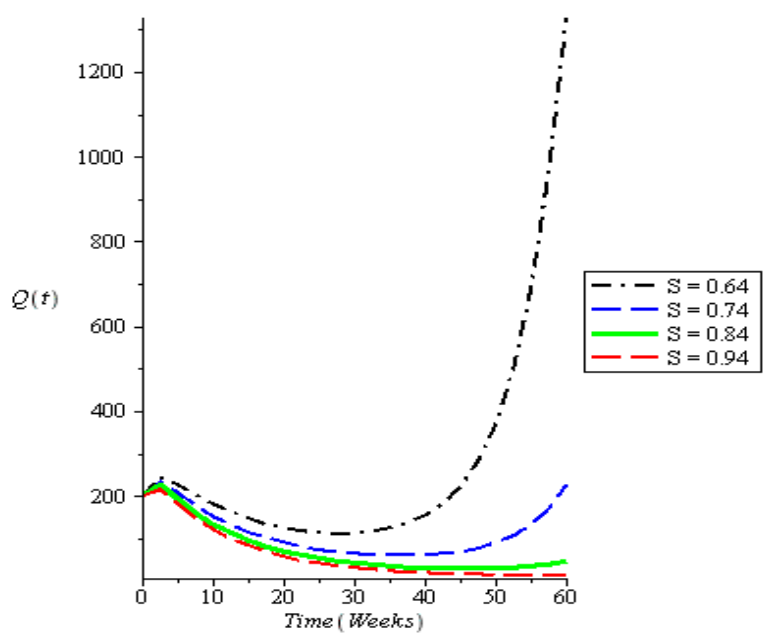

Fig. 10: Plot of the effect of social inhibition, $S$ on the hive bees infested by infectious vectors 


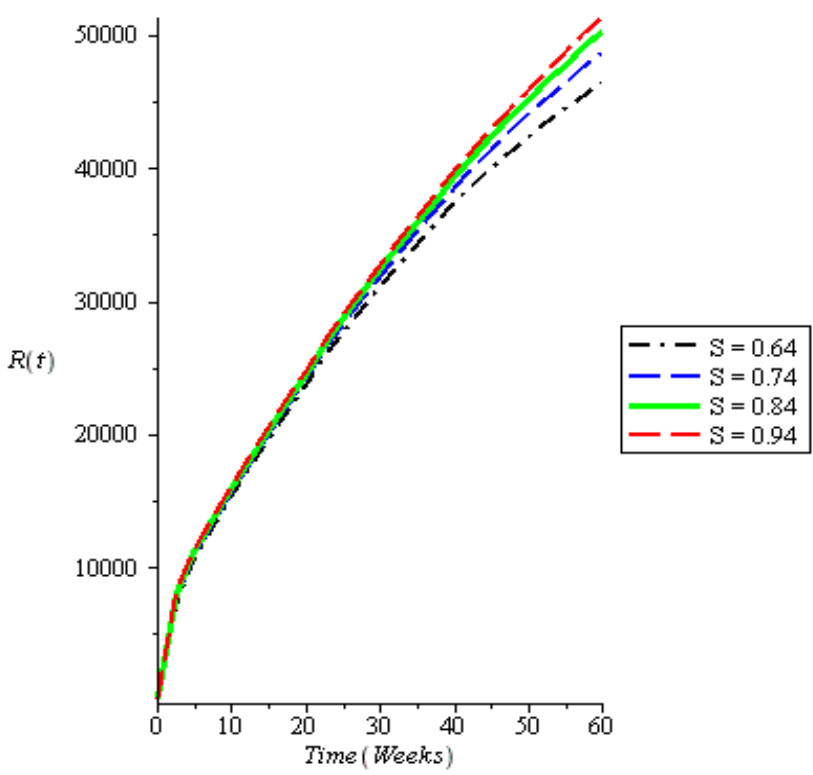

Fig. 11: Plot of the effect of social inhibition, $S$ on the recovered class

\subsection{Discussion of Results}

From the numerical simulation that was carried out on the eco-epidemiological model, it was observed from (Figure 2): the effect of transmission rate was investigated on the susceptible hive bees. The plots showed that the population of susceptible hive bees decreased with time as the transmission rate $\beta_{1}$ increased. Also, from (Figure 3): the effect of transmission rate was investigated on the plot showed that the population increased with time as the transmission rate increased.

From Figures (4 and 5): the effect of transmission rate $\beta_{2}$ was investigated on the susceptible forager bees and, the plot showed that the population of susceptible forager bees and hive bees infested by non-infectious vectors decreased with time as the transmission rate $\beta_{2}$ increased.

From Figures (6 and 7): the effect of the disinfestation rate $\alpha$ was investigated and, the plot showed that the population of hive bees infested by infectious vectors and hive bees infested by non-infectious vectors decreased with time as the disinfestation rate $\alpha$ increased.

Conversely, in Figure (8): the effect of disinfestation rate, $\alpha$ was investigated on the $R(t)$ Recovered/Removed hive bees, the plot showed that the population of $R(t)$ Recovered/Removed hive bees increased with time as the disinfestation rate $\alpha$ increased.

From Figures (9 and 10): the effect of social inhibition was investigated on the susceptible hive bees $M(t)$ and hive bees infested by infectious vectors, the plot showed that the population of susceptible hive bees and hive bees infested by infectious vectors $Q(t)$ decreased with time as the social inhibition $S$ increased.

Finally, Figure (11): the effect of social inhibition, $S$ was investigated on the $R(t)$ Recovered/Removed hive bees, the plot showed that the population of $R(t)$ Recovered/Removed hive bees undergoes some stability in their increased with time as the social inhibition $S$ increased.

\subsection{Conclusion}

In this work, an eco-epidemiological model of the honeybee with varroa mite interacting populations has been studied. Qualitative and quantitative analyses were carried out on the formulated model to provide insights into the behavior of the honeybee colony system in the presence of varroa mite disease with recovery class. The well-posedness of the model has been established by using the basic theory of positivity and boundedness solutions. Further, five equilibria (such as trivial, disease-free and infestation free, disease-free with an infestation, endemic and infestation with infectious vectors, and endemic and infestation with infectious and noninfectious vectors) were derived.

Investigation of the local stability of the equilibrium points was determined using the Jacobian matrix with the RouthHurwitz criterion. Also, global stability analysis of the equilibria was investigated. The impacts of some parameters of the model were also investigated through sensitivity analysis, revealed that the maximum growth rate of foragers, $\sigma_{1}$ and the transmission rate $\beta_{1}$ are the most sensitive parameters with positive indices, while $\sigma_{2}$ and $\alpha$ have negative indices. Moreover, the numerical simulations showed that an increase in the transmission rates $\beta_{1}$ and $\beta_{2}$ decreased the populations of the susceptible hive and forager bees, but increased the populations of hive bees infested by both infectious and non-infectious vectors.

\section{ACKNOWLEDGMENT}

The authors express thank you to the anonymous referees whose comments led to this improved paper

\section{REFERENCES}

[1] AttilaDénes and Mahmoud A. I. (2019). Global dynamics of a mathematical model for a honeybee colony infested by viruscarrying varroa mites. Journal of Applied Mathematics and computing.

[2] Betti, M.I., Wahl, L.M., Zamir, M. (2014). Effects of infection on honey bee population dynamics: a model. PLoS ONE 9(10), e110237

[3] Büchler, R., Berg, S., Le Conte, Y. (2010). Breeding for resistance to Varroa destructor in Europe. Apidologie 41(3): 393-408

[4] David K.S., Myerscough M.R., and Barron A.B., (2011). A Quantitative Model of honeybee colony population dynamics. PLoS ONE 6(4): e18491. Doi:10.1371/ Journal.pone.0018491

[5] David K.S., Barron A.B., and Myerscough M.R., (2013). Modeling Food and Population Dynamics in Honey Bee Colonies. PLoS ONE 8(5): e59084. Doi:10.1371/ Journal.pone.0059084

[6] Genersch, E., Evans, J.D., Fries, I. (2010). Honey bee disease overview. J. Invertebr. Pathol 103(Supplement): S2-S4. 
[7] Goulson, D., Nicholls, E., Botías, C., Rotheray, E.L. (2015). Bee declines are were driven by combined stress from parasites, pesticides, and lack of flowers. Science 347(6229), 1255957

[8] Ho, M.-W., Cummins, J. (2007). Mystery of disappearing honeybees. Sci. Soc. 34: 35-36

[9] Kang, Y., Blanco, K., Davis, T., Wang, Y., DeGrandi-Hoffman, G. (2016). Disease dynamics of honeybees with Varroa destructor as parasite and virus vector. Math. Biosci. 275: 71-92

[10] Mid-Atlantic Apiculture Research: The Colony and Its Organization.

[11] Ramsey, S.D., Ochoa, R., Bauchan, G., Gulbronson, C., Mowery, J.D., Cohen, A., Lim, D., Joklik, J., Cicero, J.M., Ellis, J.D., et al. (2019). Varroa destructor feeds primarily on honey bee fat body tissue and not hemolymph. Proc. Natl. Acad. Sci. 116(5): 17921801

[12] Ratti, V., Kevan, P.G., Eberl, H.J. (2017). A mathematical model of forager loss in honeybee colonies infested with Varroa destructor and the acute bee paralysis virus. Bull. Math. Biol. 79(6): 1218-
1253

[13] Ratti V., Peter G. K., and Hermann J.E. (2012). A mathematical model for population dynamics in honeybee colonies infested with Varroa destructor and the Acute Bee Paralysis Virus University of Guelph, Guelph, On, N1G 2W1, Canada

[14] Rosenkranz, P., Aumeier, P., Ziegelmann, B. (2010). Biology and control of Varroa destructor. J. Invertebr. Pathol. 103.S96-S119

[15] Smith, D.R., Villafuerte, L., Otis, G., Palmer, M.R. (2000). Biogeography of Apis cerana F. and A. nigrocincta Smith: insights from mtDNA studies. Apidologie 31(2) : 265-279

[16] Staveley, J.P., Law, S.A., Fairbrother, A., Menzie, C.A. (2014). A causal analysis of observed declines in managed honey bees (Apis mellifera). Hum. Ecol. Risk Assess. 20(2): 566-591

[17] VanEngelsdorp, D., Evans, J.D., Saegerman, C., Mullin, C., Haubruge, E., Nguyen, Watanabe, E.M. (2009). Colony collapse disorder: many suspects, no smoking gun. BioScience 58(5): 384388 